\title{
Article \\ Combined Transcriptomic Analysis and RNA Interference Reveal the Effects of Methoxyfenozide on Ecdysone Signaling Pathway of Spodoptera exigua
}

\author{
Zhixian Zhang ${ }^{1,2,+}$, Yajie Ma ${ }^{2,+}$, Xiaoyan Ma ${ }^{1,2}$, Hongyan Hu ${ }^{2}$, Dan Wang ${ }^{2}$, Xianpeng Song ${ }^{2}$, \\ Xiangliang Ren ${ }^{1,2, *}$ and Yan Ma ${ }^{1,2, *}$ \\ 1 Zhengzhou Research Base, State Key Laboratory of Cotton Biology, Zhengzhou University, \\ Zhengzhou 450001, China; zhangzhixianmhs@163.com (Z.Z.); harmani@126.com (X.M.) \\ 2 State Key Laboratory of Cotton Biology, Institute of Cotton Research, Chinese Academy of Agricultural \\ Sciences, Anyang 455000, China; yun1268486@126.com (Y.M.); huhongyan1986@163.com (H.H.); \\ nywangdan@sina.com (D.W.); sxp15294@126.com (X.S.) \\ * Correspondence: renxiangliang@caas.cn (X.R.); mayan@caas.cn or aymayan@163.com (Y.M.) \\ + Co-first authors.
}

check for updates

Citation: Zhang, Z.; Ma, Y.; Ma, X.; Hu, H.; Wang, D.; Song, X.; Ren, X.; Ma, Y. Combined Transcriptomic Analysis and RNA Interference Reveal the Effects of Methoxyfenozide on Ecdysone Signaling Pathway of Spodoptera exigua. Int. J. Mol. Sci. 2021, 22, 9080. https://doi.org/10.3390/ijms22169080

Academic Editor: Marcello Iriti

Received: 14 July 2021

Accepted: 19 August 2021

Published: 23 August 2021

Publisher's Note: MDPI stays neutral with regard to jurisdictional claims in published maps and institutional affiliations.

Copyright: (c) 2021 by the authors. Licensee MDPI, Basel, Switzerland. This article is an open access article distributed under the terms and conditions of the Creative Commons Attribution (CC BY) license (https:// creativecommons.org/licenses/by/ $4.0 /)$.

\begin{abstract}
Spodoptera exigua is a worldwide pest afflicting edible vegetables and has developed varying levels of resistance to insecticides. Methoxyfenozide (MET), an ecdysteroid agonist, is effective against lepidopteran pests such as S. exigua. However, the mechanism of MET to S. exigua remains unclear. In this study, we analyzed the expression patterns of genes related to the ecdysone signaling pathway in transcriptome data treated with sublethal doses of MET and analyzed how expression levels of key genes affect the toxicity of MET on S. exigua. Our results demonstrated that 2639 genes were up-regulated and 2512 genes were down-regulated in S. exigua treated with $\mathrm{LC}_{30}$ of MET. Of these, 15 genes were involved in the ecdysone signaling pathway. qPCR results demonstrated that ecdysone receptor $A(E c R A)$ expression levels significantly increased in S. exigua when treated with different doses of MET, and that the RNAi-mediated silencing of EcRA significantly increased mortality to $55.43 \%$ at $72 \mathrm{~h}$ when L3 S. exigua larvae were exposed to MET at the $\mathrm{LC}_{30}$ dose. Additionally, knocking down EcRA suppressed the most genes expressed in the ecdysone signaling pathway. The combination of MET and $\operatorname{ds} E c R A$ affected the expression of $E 74$ and enhanced the expression of TREA. These results demonstrate that the adverse effects of sublethal MET disturb the ecdysone signaling pathway in S. exigua, and EcRA is closely related to MET toxic effect. This study increases our collective understanding of the mechanisms of MET in insect pests.
\end{abstract}

Keywords: Spodoptera exigua; methoxyfenozide; toxicity; ecdysone receptor A; mortality

\section{Highlights}

Five genes were up-regulated and 10 genes were down-regulated in Spodoptera exigua treated with $\mathrm{LC}_{30}$ of Methoxyfenozide, all of which were involved in the ecdysone signaling pathway.

The expression of $E c R A$ was up-regulated as treatments of Methoxyfenozide on S. exigua increased.

Knockdown of EcRA expression by RNAi dramatically increases the sensitivity of S. exigua to Methoxyfenozide.

Knockdown of EcRA expression affects the expression of the ecdysone signaling pathway genes in Methoxyfenozide-treated S. exigua.

\section{Introduction}

Spodoptera exigua (Lepidoptera, Noctuidae) is a polyphagous pest. It attacks important cultivated and industrial crops, including cotton and sugar beets, resulting in significant 
losses [1]. Therefore, controlling S. exigua is important for agricultural production, and insecticides can be used for this purpose [2-4]. Traditional chemical insecticides are the primary method of controlling S. exigua [2-4]. However, the doses of traditional chemical controls increase with time and can damage the crops [5]. Integrated Pest Management (IPM) is a widely used method of controlling insect pests, while Insect Growth Regulators (IGRs) play a pivotal role in IPM [6]. Compared with traditional chemical controls, IGRs have specific advantages: they produce less residue, are easily degradable, and can induce insect molting which results in death [7].

IGRs contain Juvenile Hormone agonists (JH), ecdysteroid agonists (20E), and Chitin Biosynthesis Inhibitors (CSI). 20E is a molting hormone and combines with the ecdysone receptor (EcR) and ultraspiracle (USP), turning into a compound [8]. This compound triggers the expression of the 20E response gene. The research literature shows that ecdysone signaling pathway involves in $E c R, U S P$, and several other ecdysone-responsive genes, including the ecdysone-induced protein $74 E F$ (E74), nuclear hormone receptor HR3 (HR3), hormone receptor 4 (HR4), hormone receptor FTZ-F1 (FTZ-F1), hormone receptor HR38 (HR38), and chitin biosynthesis pathway genes [9-12]. The chitin biosynthesis pathway contains Trehalose 6-phosphate (TPS), Trehalase (TRE), Hexokinase (HK), Glucose-6-phosphate isomerase (G6PI), Glutamine fructose-6-phosphate aminotransferase (GFAT), Phosphoacetyl Glucosamine Mutase (PGM), N-acetyltransferase UDP-N-acetylglucosamine Pyrophosphorylase (UAP), and Chitin Synthase (CHS), all of which have been found in several insect species [13-16].

In Lepidoptera, the prothoracicotropic hormone (PTTH)-stimulated ecdysteroidogenesis and larval growth are involved in two ways, mitogen-activated protein kinase (MAPK) and phosphoinositide 3-kinase/protein kinase B/target of rapamycin (PI3K/Akt/TOR) pathways $[14,17,18]$. Ecdysone is subsequently activated to $20-\mathrm{OH}$-ecdysone in peripheral tissues [17]. 20E binds to a heterodimer of the EcR and USP, and the 20E-EcR/USP complex controls the transcription of target genes [8]. EcR is an ecdysone receptor and has been isolated from many insect species. Helicoverpa armigera that were fed Escherichia coli expressing amiRNA-HaEcR exhibited developmental defects and high rates of mortality [12]. The suppression of $\operatorname{SeEcR}$ influenced developmental duration, pupae formation, adult eclosion, and the chitin content of S. exigua [19]. To elucidate the role of EcR in physiological processes, the relationship between $E c R$ and insecticides has been reported. In Conopomorpha sinensis, EcR expression was changed by chlorbenzuron [20]. Treating $S$. exigua with tebufenozide or RH-5849 demonstrated that ecdysone agonists binding with $E c R$ [21]. After treatment with Methoxyfenozide (MET), EcR expression was enhanced, and subsequently impaired fecundity [22]. MET is an ecdysteroid agonist and can combine with EcR compounds, leading to lethal molting [23]. Therefore, some studies assessed the toxicity of S. exigua treated with MET [24,25]. However, few studies have assessed how MET affects the ecdysone signaling pathway genes and the mechanism for MET toxicity in S. exigua.

In this study, the transcriptome of S. exigua fed with MET were sequenced using an Illumina TruseqTM RNA Sample Prep Kit. We determined that the ecdysone signaling pathway genes associated with S. exigua fed with MET. Using quantitative real-time PCR (qPCR), identified the gene expression change and used RNAi to assess the role that $E c R A$ involve in increasing S. exigua sensitivity to MET. We also assessed the expression patterns of ecdysone signaling pathway gene changes in MET and RNAi treated larvae. These results reveal different doses of MET affects gene expression on ecdysone signaling pathway, exhibiting that knocking down EcRA impacts mortality of MET and the expression of ecdysone signaling pathway genes of $S$. exigu. This study attempts to determine the interaction of EcRA and MET on S. exigua, and further explore the action mechanisms of MET. 


\section{Results}

\subsection{Transcriptome Data Analysis}

In order to identify the genes involved in the ecdysone signaling pathway, transcriptome sequencing analysis was used to sequence the transcriptomes of $S$. exigua larvae in MET and control groups. Quality control was performed on the original sequencing data to obtain high-quality data and conduct statistical analyses (Table 1). The transcriptome sequences were connected, filtered, and optimized using TransRate and Trinity. The two group transcriptome sequencing results were completed, after which the bioinformatics analysis was performed. The relativity of the sample data indicated that the results of the transcriptome sequencing were viable. The UniGenes and Transcripts number were successfully annotated in S. exigua (Figure 1a). Spodoptera litura had the highest homology compared with S. exigua (Figure 1b).

Table 1. Date control statistics of transcriptome of S. exigua.

\begin{tabular}{|c|c|c|c|c|c|c|c|c|}
\hline Sample & Raw Reads & Raw Bases & Clean Reads & Clean Bases & Error Rate (\%) & Q20 (\%) & Q30 (\%) & GC Content $(\%)$ \\
\hline Control group 1 & $44,923,180$ & $6,783,400,180$ & $44,335,152$ & $6,636,272,205$ & 0.0236 & 98.66 & 95.48 & 46.75 \\
\hline Control group 2 & $46,298,736$ & $6,991,109,136$ & $45,854,460$ & $6,864,878,635$ & 0.0230 & 98.88 & 96.17 & 49.38 \\
\hline Control group 3 & $46,272,298$ & $6,987,116,998$ & $45,733,494$ & $6,851,482,844$ & 0.0231 & 98.85 & 96.06 & 49.08 \\
\hline $\operatorname{MET}\left(\mathrm{LC}_{30}\right) 1$ & $48,311,340$ & $7,295,012,340$ & $47,908,742$ & $7,186,907,127$ & 0.0227 & 99.01 & 96.49 & 49.14 \\
\hline $\operatorname{MET}\left(\mathrm{LC}_{30}\right) 2$ & $49,407,600$ & $7,460,547,600$ & $48,903,418$ & $7,321,874,857$ & 0.0229 & 98.93 & 96.28 & 49.00 \\
\hline $\operatorname{MET}\left(\mathrm{LC}_{30}\right) 3$ & $48,680,700$ & $7,350,785,700$ & $48,177,980$ & $7,222,758,714$ & 0.0229 & 98.91 & 96.21 & 48.38 \\
\hline
\end{tabular}
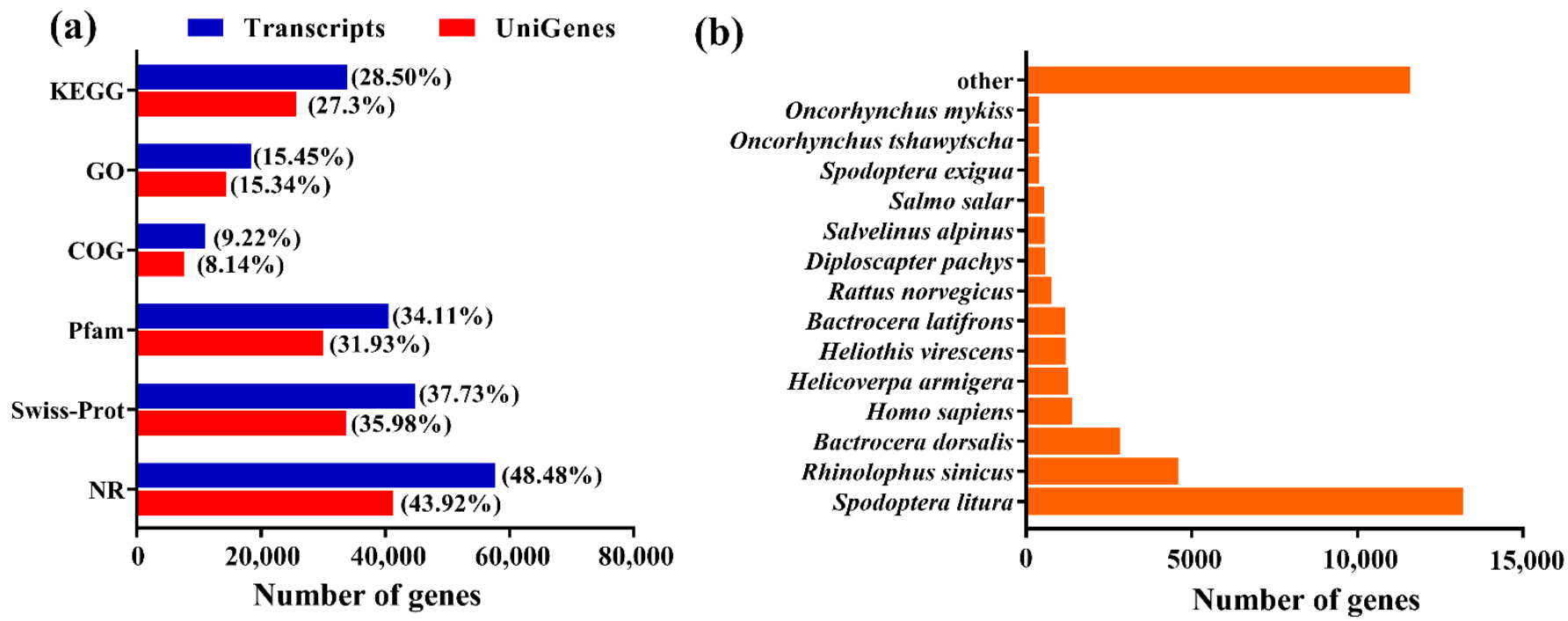

Figure 1. Gene annotation and homology search. (a) The number of genes using six databases. NR, Swiss-Prot, Pfam, COG, GO, and KEGG. (b) Homology search of Illumina sequences matching with the NR database. 18,935 NR-annotated transcripts sequences were analyzed for species distribution.

The total number of transcripts and UniGenes were 118,834 and 93,874. After filtering, the average error rate located with $0.02 \sim 0.03 \%$. The results show that $>98 \%$ of each sample had a quality score of Q20, and $>95 \%$ of each sample had a quality score of Q30. The GC content of the S. exigua were more than $49 \%$ (control group) and $49 \%$ (MET).

The functional distribution of differentially expressed genes (DEGs) in S. exigua was determined by comparing its transcriptomes in response to MET. Compared with the control group, 2639 genes were up-regulated and 2512 genes were down-regulated (Figure 2a). According to the GO terms, 3798 UniGenes were divided into three categories (biological processes, molecular functions, and cellular components) containing 20 variety classes. Catalytic activity (167), binding (104), and metabolic processes (88) contained the most UniGenes in the three categories (Figure 2b). By comparing the up-regulated and down- 
regulated GO enrichment, it was apparent that nearly all of them in S. exigua were more up-regulated than down-regulated after exposure to the sublethal dose of MET (Figure S1). KEGG enrichment showed that 20 KEGG pathways were assigned to signification enrichment (Figure 2c). There were 15 genes involved in the ecdysone signaling pathway (Table 2). In qPCR, EcRA, USP, and HR3 were up-regulated and E74, HR4, FTZ-F1, and HR38 were down-regulated. The qPCR results were compared with the RNA-seq analysis, indicating that the RNA-seq results were reliable (Figure S2).

(a)

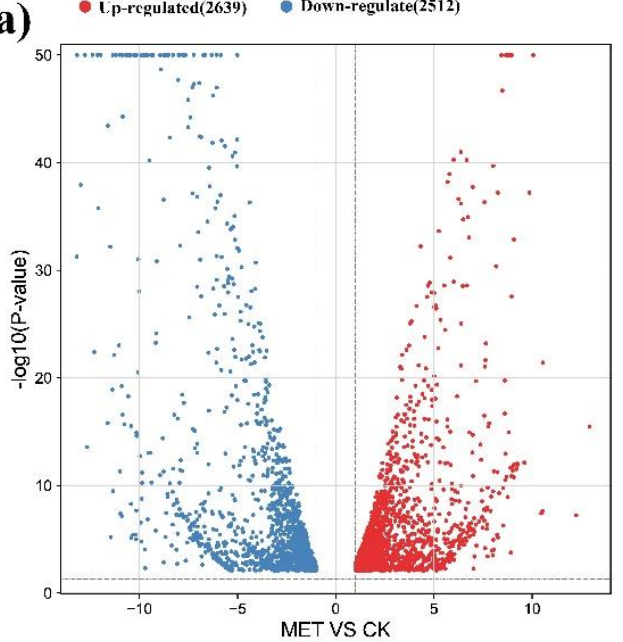

(c)

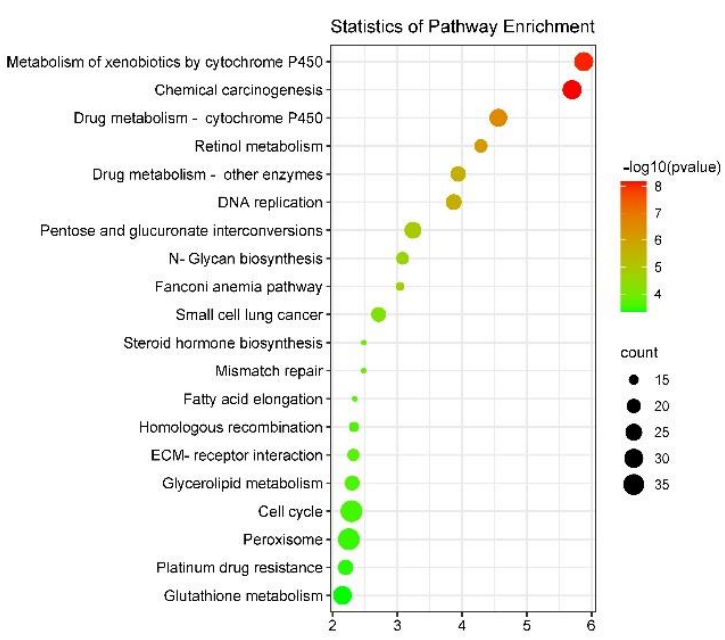

GO annotations

(b)

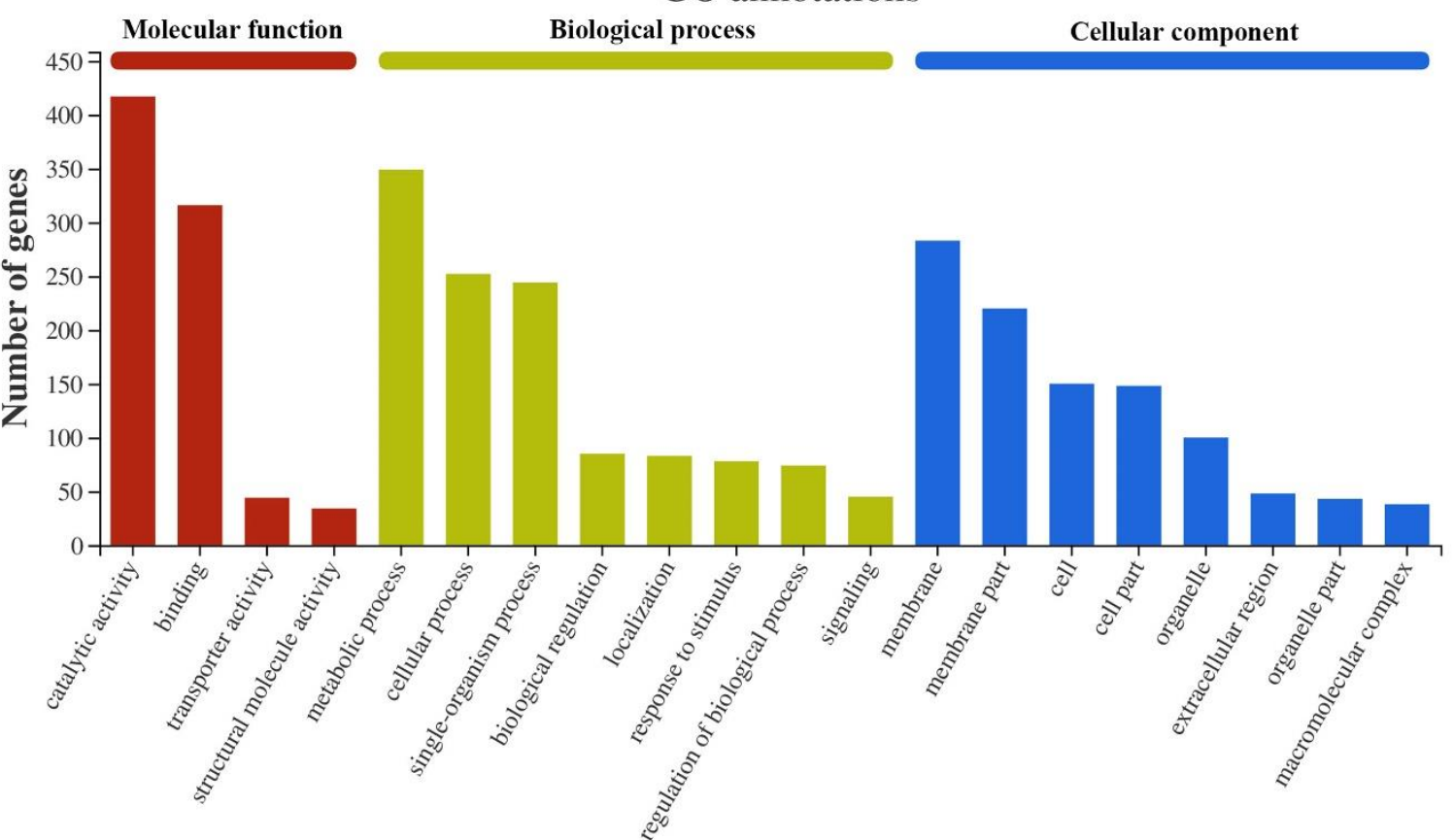

GO term

Figure 2. Function annotation and enrichment of DEGs. (a) Volcano plot of differentially expressed genes of S. exigua fed with MET (red spots represent significantly up-regulated genes; green spots represent significantly down-regulated genes). (b) GO function annotation analysis of S. exigua fed with MET (metabolic process under biological process had the primary number of UniGenes. The catalytic activity and binding under the molecular function were the most dominant. The membrane under the cellular component were the biggest group). (c) The most enriched KEGG pathways of S. exigua after exposure to a sublethal concentration of MET. 
Table 2. Ecdysone signaling pathway of DEGs.

\begin{tabular}{|c|c|c|c|c|c|}
\hline UniGenes & Gene Bank & Homologous Genes & $\log _{2} \mathrm{FC}$ & Padjust & Organisms \\
\hline TRINITY_DN68411_c0_g1 & ACD39740.1 & ultraspiracle protein (USP) & 1.43053001 & 0.000710809 & S. exigua \\
\hline TRINITY_DN68123_c0_g1 & XP_022816977.1 & probable nuclear hormone receptor HR3 isoform X6 (HR3) & 1.319971145 & 0.407707359 & S. litura \\
\hline TRINITY_DN67384_c0_g4 & GU296540.1 & ecdysone receptor $A(E c R A)$ & 0.511687018 & 0.509814187 & S. exigua \\
\hline TRINITY_DN66888_c3_g2 & XP_022822951.1 & nuclear hormone receptor FTZ-F1 beta (FTZ-F1) & -0.85620132 & 0.101532649 & S. litura \\
\hline TRINITY_DN35412_c0_g1 & XM_022967707.1 & PREDICTED: ecdysone-induced protein 74EF (E74) & -0.86981036 & 1 & S. litura \\
\hline TRINITY_DN62913_c0_g1 & XP_021187825.1 & hormone receptor 4 isoform $\mathrm{X} 1$ (HR4) & -3.19753521 & $8.62 \times 10^{-9}$ & H. armigera \\
\hline TRINITY_DN68583_c0_g4 & XP_022820857.1 & probable nuclear hormone receptor HR38 (HR38) & -6.28201224 & $3.90 \times 10^{-40}$ & S. litura \\
\hline TRINITY_DN65723_c0_g2 & ACV97159.1 & glucose-6-phosphate isomerase (G6PI) & 1.468813444 & 0.000855695 & S. exigua \\
\hline TRINITY_DN66137_c0_g2 & ABY86218.1 & trehalase-1 (TREA) & 0.83888701 & 0.486117572 & S. exigua \\
\hline TRINITY_DN67124_c0_g6 & ABM66814.2 & trehalose 6-phosphate synthase (TPS) & -0.15968318 & 0.840417477 & S. exigua \\
\hline TRINITY_DN63532_c0_g3 & XP_022834981.1 & hexokinase type 2 isoform $\mathrm{X} 6(\mathrm{HK})$ & -0.54980536 & 0.337950088 & S. litura \\
\hline TRINITY_DN68071_c2_g3 & XP_022827210.1 & Phosphoacetyl glucosamine mutase (PGM) & -0.81471571 & 0.132596543 & S. litura \\
\hline TRINITY_DN65476_c0_g2 & AAZ03545.1 & chitin synthase A (CHSA) & -1.99147489 & $2.42017 \times 10^{-8}$ & S. exigua \\
\hline TRINITY_DN66504_c1_g4 & XP_022821069.1 & glutamine-fructose-6-phosphate aminotransferase (GFAT) & -4.07457569 & $3.01573 \times 10^{-29}$ & S. litura \\
\hline TRINITY_DN67977_c1_g2 & ACN29686.1 & UDP-N-acetylglucosamine pyrophosphorylase (UAP) & -5.02829161 & $1.86507 \times 10^{-40}$ & S. exigua \\
\hline
\end{tabular}

\subsection{Sublethal Doses of MET Induction on Ecdysone Signaling Pathway}

Different doses $\left(\mathrm{LC}_{10}, \mathrm{LC}_{30}\right.$, and $\left.\mathrm{LC}_{50}\right)$ of MET were used to examine the dosedependent effects in ecdysone signaling pathway gene expression in S. exigua. EcRA, USP, and HR3 were positively up-regulated and E74, HR4, FTZ-F1, and HR38 were down-regulated after the larvae were treated with different doses of MET. Notably, the up-regulation of $E c R A$ increased to 10.85-fold under $\mathrm{LC}_{10}$ treatment, 14.02-fold under $\mathrm{LC}_{30}$ treatment, and 14.81-fold under $\mathrm{LC}_{50}$ treatment (Figure 3). TPS and TREA were up-regulated and HK, G6PI, GFAT, UAP, and CHSA were down-regulated after S. exigua were treated with different doses of MET. The up-regulation of TREA increased 1.65-fold under $\mathrm{LC}_{10}$ treatment, 2.66-fold under $\mathrm{LC}_{30}$ treatment, and 4.28-fold under $\mathrm{LC}_{50}$ treatment (Figure 3).

\subsection{Down-Regulation of EcRA Increase the Mortality of MET to S. exigua}

In order to explore the role of EcRA in toxicity of MET, we knocked down EcRA and interfering efficiency in epidermis was examined. Our results showed that the relative transcription levels of $E c R A$ did not significantly change after treatment with MET at $12 \mathrm{~h}$. However, the transcripts of $E c R A$ were attenuated from $24 \mathrm{~h}$ to $72 \mathrm{~h}$ after RNAi (Figure $4 \mathrm{a}$ ). The lowest expression level of EcRA was found at $48 \mathrm{~h}$, with an 0.03 -fold to the dsGFP. In addition, a nonsignificant benefit in mortality was observed when compared with dsGFP after the dsEcRA was injected at $24 \mathrm{~h}, 48 \mathrm{~h}$, and $72 \mathrm{~h}$ (not shown). Knock down of $E c R A$ in L3 larvae resulted in increased mortality. Approximately $55.43 \%$ of MET-dsEcRA treated larvae at the $\mathrm{LC}_{30}$ dose of MET (Figure $4 \mathrm{~b}$ ).

\subsection{The Expression of Ecdysone Signaling Pathway Genes Are Regulated by MET-dsEcRA}

We collected the survival larvae treated with MET-dsEcRA at $72 \mathrm{~h}$, the relative transcription levels of ecdysone signaling pathway genes were checked by qPCR. It was found that knock down of $E c R A$ could influence other genes in the ecdysone signaling pathway of $S$. exigua (Figure 5a). The expression levels of HR3, HR4, and HR38 could be downregulated by 0.34 -fold, 0.36 -fold, and 0.37 -fold, respectively, after the larvae treated with MET-dsEcRA. The expression levels of $E 74$ were up-regulated 2.15-fold, while there was no change in gene expression of USP and FTZ-F1. Moreover, down-regulation of EcRA affected expression of chitin biosynthesis pathway genes. The expression levels of G6PI, $U A P$, and CHSA were down-regulated by 0.42 -fold, 0.26 -fold, and 0.48 -fold, respectively (Figure 5b). 

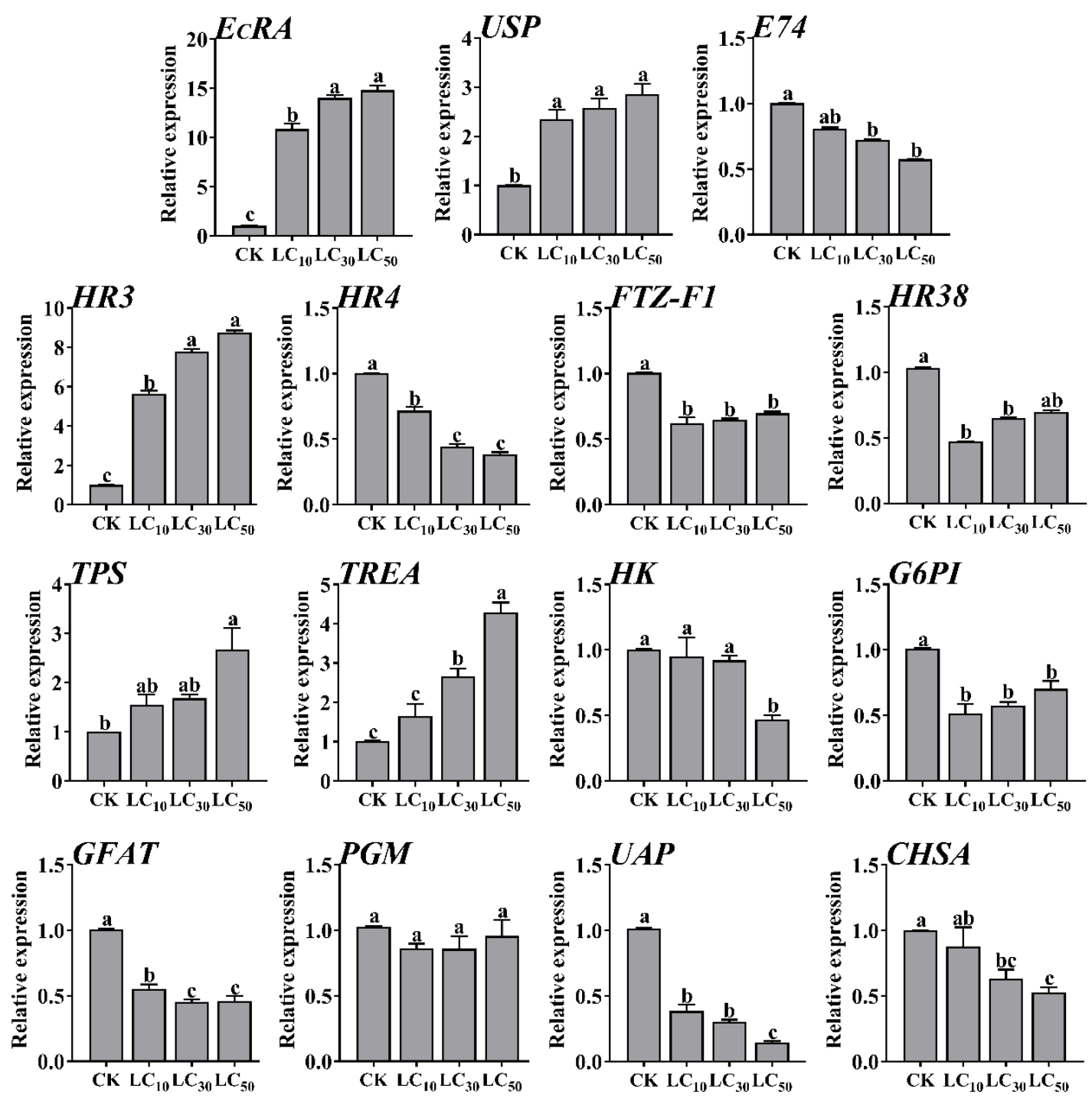

Figure 3. Relative expression levels of ecdysone signaling pathway genes in S. exigua under MET treatment. Relative expression levels of ecdysone signaling pathway in L3 larvae were examined $72 \mathrm{~h}$ after exposure to the control treatment DEPC water $(\mathrm{CK})$ or MET at three different dosages $\left(\mathrm{LC}_{10}, \mathrm{LC}_{30}\right.$, and $\left.\mathrm{LC}_{50}\right)$. The bars represent the average ( $\left.\pm \mathrm{SE}\right)$ of biological repeats. Different letters above the bars indicate significant differences $(p<0.05)$, based on one-way ANOVA and at least significant difference test.

(a)

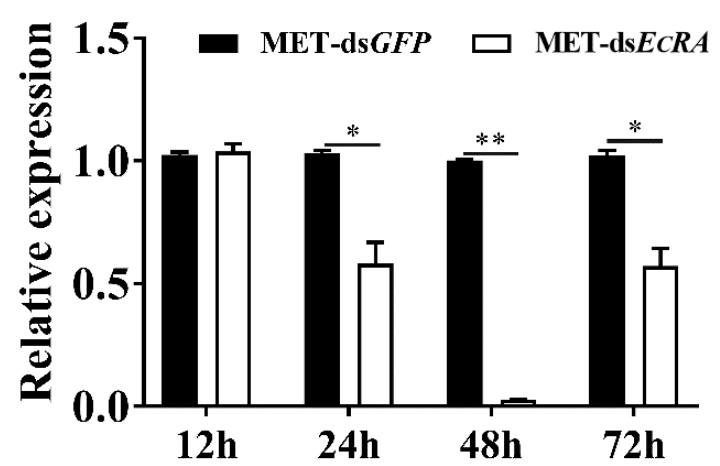

(b)

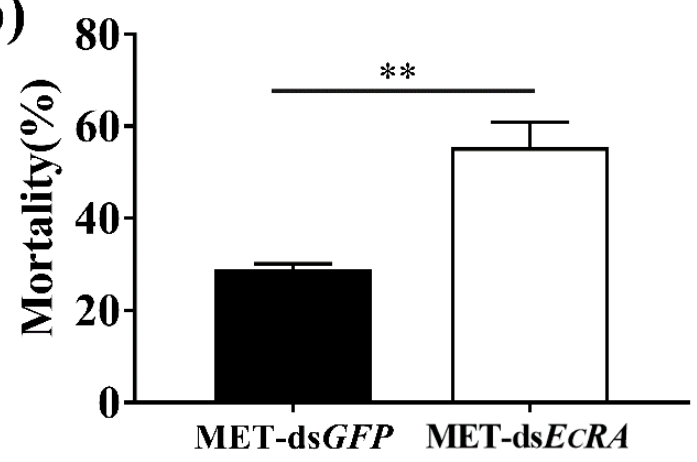

Figure 4. Knockdown of $E c R A$ increases the sensitivity of S. exigua to MET. (a) Relative expression of $E c R A$; (b) mortality (\%) of the dsEcRA-treated S. exigua at $72 \mathrm{~h}$ after MET treatment. The columns represent averages, with vertical lines indicating SE. Significant differences were calculated by Student's $t$-test $\left({ }^{*} p<0.05 ;{ }^{* *} p<0.01\right)$. 
(a)

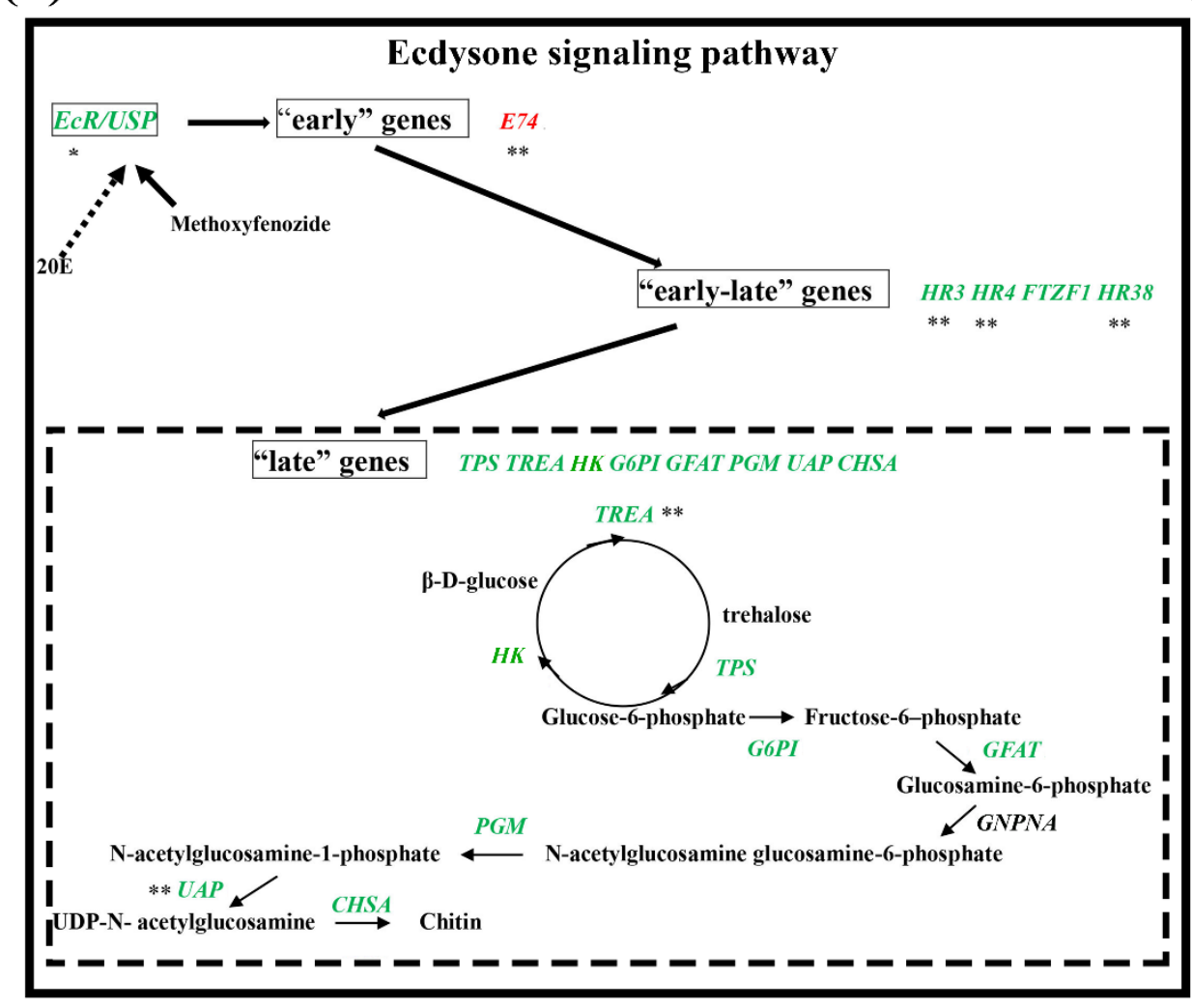

(b)

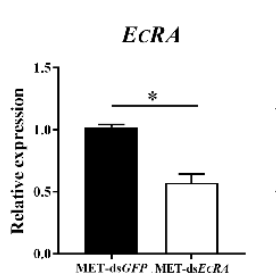

HR3

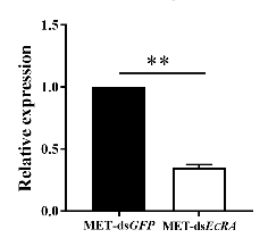

$T P S$

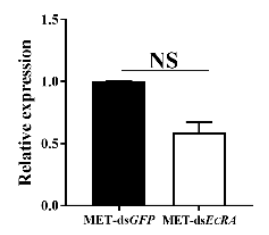

GFAT

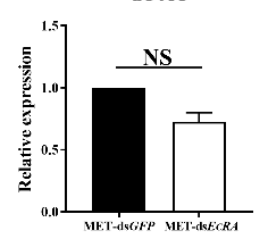

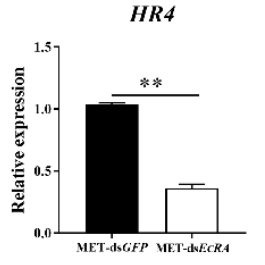

TREA

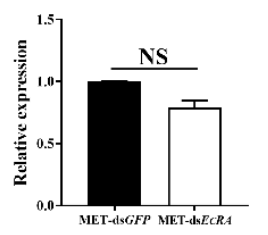

PGM

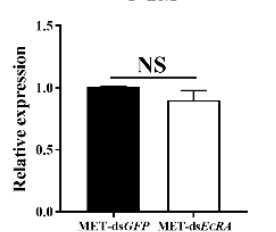

USP

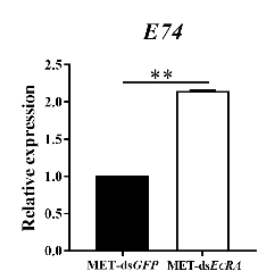

FTZ-F1

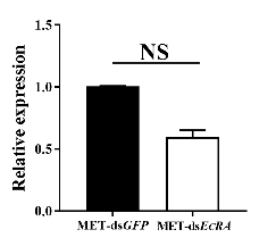

HK

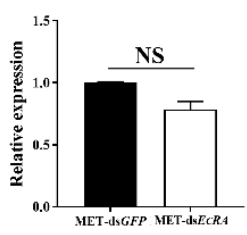

UAP

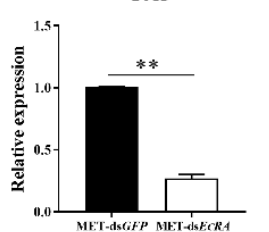

HR38

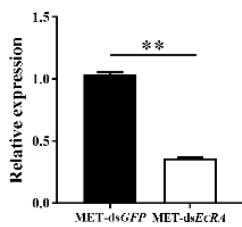

G6PI

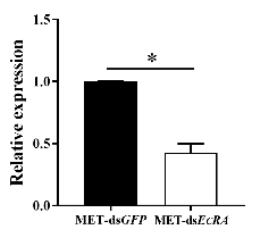

CHSA

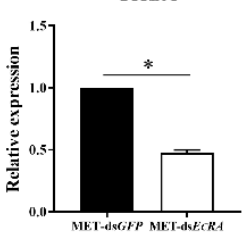

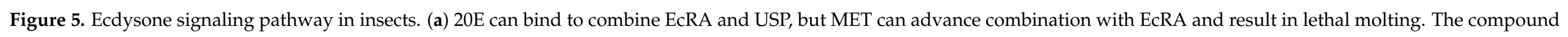

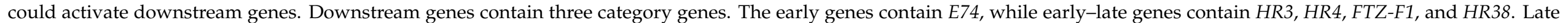

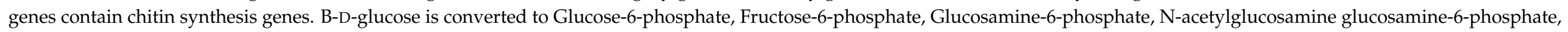

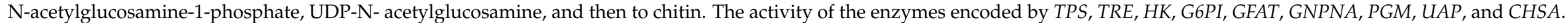

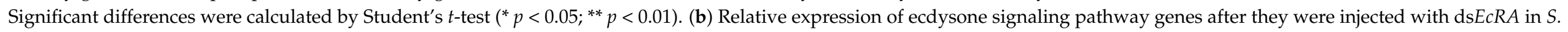

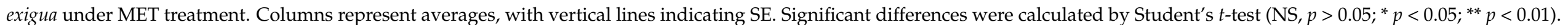




\section{Discussion}

S. exigua (Lepidoptera, Noctuidae) is a polyphagous pest. It attacks high value cultivated and industrial crops, including cotton and sugar beets [1]. Therefore, some studies assessed the toxicity of S. exigua treated with MET [24,25]. However, there are few studies assessing the relationship between MET toxicity and the role of genes in S. exigua. In this study, we analyzed S. exigua after it was treated with MET to assess the role of genes involved in the toxicity of MET. The result of the transcriptome sequencing, DEGs, qPCR, RNAi, and bioassay analysis exhibited that sublethal doses of MET could adversely affect the ecdysone signaling pathway, and knock down of EcRA increased the mortality of MET to S. exigua.

Transcriptome sequencing is used to identify the relationship between insecticides, pests, and key genes involved in pest control. Analysis of the transcriptome sequencing of Lufenuron against Spodoptera frugiperda provided several genes for defensive mechanisms [26]. We found 15 genes in the ecdysone signaling pathway of S. exigua using transcriptome sequencing. This lays a foundation for studying the relationship between insecticides and ecdysone signaling pathway genes. EcR and SfUSP-2 were significantly induced by the hormone agonist MET [27]. The expression of PxTre increased in flubendiamide [28]. The expression of HK, G6PI2, GFAT, PAGM1, and UAP genes decreased significantly after the injection of validamycin [29], while mRNA levels of ChsA dramatically decreased in response to chlorantraniliprole [30]. MET affects development and changes the expression of the ecdysone signaling pathway of S. exigua [25]. Previous studies also have demonstrated that MET affected the expression of $H$. armigera 20E-related genes [31]. Our results confirmed the inductive effect of MET on the ecdysone signaling pathway. This conclusion is similar to the effect that halofenozide has on 20E-response genes in Leptinotarsa decemlineata, as halofenozide can activate detoxification, and the ability of agonists to regulate the expression of the 20E-response genes [32]. It has been shown that sublethal doses of MET lowered the intrinsic 20E titer [31]; therefore, MET affects larval development due to the change it induces in the intrinsic $20 \mathrm{E}$ titer. In our research, $E c R A$ was significantly regulated in three different sublethal doses. Interestingly, Plutella xylostella was treated with $\mathrm{LC}_{25}$ and $\mathrm{LC}_{50}$ of fufenozide for $48 \mathrm{~h}$, and $P x E c R-B$ expression was downregulated [33]. EcR expression trends in the two species of Lepidoptera differed following treatment with the same kind of insecticides. Hence, we identified the EcRA gene as the primary research objective after treating S. exigua with MET. However, little is known about the interaction relationship between EcRA and MET.

$E c R A$ was an important gene in the ecdysone signaling pathway, and knock down of EcRA in S. exigua increased the sensitivity to MET. Similarly, miR-189942 suppressed $P x E c R-B$ expression and influenced the tolerance of $P$. xylostella to fufenozide [33]. In this study, we used an injection RNAi technique to determine that the expression of EcRA significantly decreased after $\mathrm{ds} E c R A$ injection compared with the control. Moreover, we applied gene silencing followed bioassay to evaluate the role of $E c R A$ in the MET toxicity to S. exigua. After the $E c R A$ was knocked down, the results of the bioassay showed that mortality of $S$. exigua was increased when exposed to MET. It indicates that $E c R A$ involves in the detoxification of MET. Overall, these findings indicate that $E c R A$ plays an important role in regulating the toxicity of MET, but that crosstalk between the MET and dsEcRA treatments for the genes in the ecdysone signaling pathway requires further study.

In this study, knockdown of EcRA influenced the expression of genes in the ecdysone signaling pathway. Similarly, the mRNA level of 20E signaling genes was significantly changed after the knockdown of $E c R$ [34-38]. However, the expression of E74 differed from previous research. There are two possible reasons for this. First, Leptinotarsa decemlineata and Henosepilachna vigintioctopunctata belong to the order Coleoptera, while S. exigua belongs to the order Lepidoptera, and the structure and composition could differ among the three pests. Second, MET affects the expression of E74. After the injection of dsEcRcom, the chitin contents in the cuticle significantly decreased [14]. In our research, the expressions 
of G6PI, UAP, and CHSA significantly changed in S. exigua treated with MET-dsEcRA. The expression levels of SeTre-1, SeG6PI, SeUAP, and SeCHSA were significantly reduced when $\mathrm{dsEcRcom}$ was injected at the larval stage of $S$. exigua [14].

Overall, our study reveals the gene expression of the ecdysone signaling pathway in $S$. exigua treated with different doses of MET, and that knocking down EcRA affects mortality of MET and the ecdysone signaling pathway. These results suggest that EcRA, as an important gene in ecdysone signaling pathway, plays an important role of totoxic process of MET to the target pest. Therefore, our findings offer a deep insight into interaction of $E c R A$ and MET, and the action mechanisms of MET.

\section{Materials and Methods}

\subsection{Chemicals}

The insecticide used in this study was Methoxyfenozide (MET) (97\% purity), which was provided by Anyang Quanfeng Biotechnology Co., Ltd. (Quanfeng Biotechnology Co., Ltd, Anyang, China). All compounds were technical grade materials, and stock solutions of $100 \mathrm{mg} / \mathrm{L}$ of technical grade materials were prepared using acetone as the solvent [23].

\subsection{Insect Rearing}

S. exigua larvae were collected from the cotton fields of Anyang, Henan, China, and had been raised for at least 30 generations in a greenhouse before the bioassay [39]. The larvae were maintained at $(28 \pm 1){ }^{\circ} \mathrm{C}$ with a photoperiod of $16: 8$ (L:D) $\mathrm{H}$ and $70 \%$ relative humidity. Adults were fed with a $10 \%(w / v)$ honey solution.

\subsection{Sample Collection and RNA Isolation}

The MET was dissolved in acetone and diluted with DEPC water to the desired concentration. L3 larvae were starved $4 \mathrm{~h}$ and placed into 24 orifice tissue culture plates, containing the artificial diet, via the drug-membrane method. A total of $100 \mu \mathrm{L}$ different sublethal doses of MET $\left(\mathrm{LC}_{10}=7.00 \mathrm{ng} / \mathrm{cm}^{2}, \mathrm{LC}_{30}=21.00 \mathrm{ng} / \mathrm{cm}^{2}, \mathrm{LC}_{50}=38.20 \mathrm{ng} / \mathrm{cm}^{2}\right)$ was added to every orifice, and $100 \mu \mathrm{L}$ of DEPC water was used as a control group [23]. The feed conditions of $S$. exigua were not changed.

After $72 \mathrm{~h}$ of MET induction, samples of the L4 larvae were taken from all treatments. Larvae were fixed and dissected, after which the cuticles were collected with tweezers. The cuticles were cleaned and placed in a $0.7 \%$ saline solution. The filter paper absorbed the residual saline from the cuticle. Biological samples were performed in triplicate, with each comprising one $\mathrm{L} 4$ larva cuticle that was released in a $1.5 \mathrm{~mL}$ tube. Total RNA ( $\mathrm{LC}_{10}$, $\mathrm{LC}_{30}, \mathrm{LC}_{50}$, and DEPC water) was isolated from the cuticle tissues using TRIzol Reagent (Invitrogen, Carlsbad, CA, USA) according to the manufacturer's instructions. Total RNA was stored at $-80^{\circ} \mathrm{C}$.

\subsection{Transcriptome Data Analysis}

Approximately $1 \mu \mathrm{g}$ of total RNA ( $\mathrm{LC}_{30}$ and DEPC water), obtained as described above, were isolated using Poly (A) mRNA with poly-T oligo attached magnetic beads (Invitrogen). Total RNA was sent to MAJORBIO CLOUD for cDNA library construction and transcriptome sequencing. The library was constructed using an Illumina TruseqTM RNA sample prep kit and the software was used to assemble all of the clean data from scratch. The reads were decomposed, after which we built the k-mer (default $k=25$ ), selected the seed k-mer, and extended both sides to form a contig. Overlapping contigs were clustered to form components, and each component became a set of possible representations of mutable shear isoforms or homologous genes. Each component had a corresponding DE Bruijn Graph. The DE Bruijn Graph of each component was simplified to output the full-length transcript of the variable-shear subtype, and the transcript corresponding to the homologous gene was combed to obtain the splicing result file. All UniGenes and transcripts obtained by transcriptome assembly were compared with six databases (NR, 
Swiss-prot, Pfam, COG, GO, and KEGG), with results providing statistics on the annotation and KEGG of each database.

The RSEM program is a tool used to estimate differentially expressed genes. The FPKM (Fragments per Kilobases per Million) method reduces the influence of differences in gene length and sequencing volume on the calculated gene expression, which can be directly used to compare differences in gene expression between different samples. Raw counts were statistically analyzed using the DESeq2 software to obtain the UniGenes and Transcripts, and compare differences in expression between the groups based on certain screening conditions.

To identify the ecdysone signaling pathway genes in S. exigua, we compiled all the known genes involved in this pathway based on reports on S. exigua and other insects and sequenced them based on their functional annotation in the transcriptome database $[19,40,41]$. Primers were designed according to homologous species sequences. The high amplification efficiency and melting curve are effective at confirming primers (Table S1).

\subsection{Sublethal Doses of MET Induction}

To investigate sublethal doses of MET induction, approximately $500 \mathrm{ng}$ of total RNA ( $\mathrm{LC}_{10}, \mathrm{LC}_{30}, \mathrm{LC}_{50}$, and DEPC water), prepared according to the above section, were selected to synthesize cDNA. The cDNA was synthesized from total RNA using Evo MMLV Reverse Transcriptase Kit (AG, Changsha, China). The cDNA synthesized mixture $(10 \mu \mathrm{L})$ contained $500 \mathrm{ng}$ total RNA, $1 \mu \mathrm{L}$ gDNA Clean Reagent, $2 \mu \mathrm{L} 5 \times$ gDNA Clean Buffer, and RNase Free water to $10 \mu \mathrm{L}$. The reaction conditions were $42^{\circ} \mathrm{C}$ for $2 \mathrm{~min}$, and $4{ }^{\circ} \mathrm{C}$ for $10 \mathrm{~min}$. The mix of step one contained $1 \mu \mathrm{L}$ Evo M-MLV RTase Enzyme Mix, $5 \mu \mathrm{L}$ $5 \times$ RTase Reaction Buffer Mix I, $1 \mu \mathrm{L}$ RT Primer MIX, and $4 \mu \mathrm{L}$ RNase Free water to $20 \mu \mathrm{L}$. The cDNA was synthesized following these cycling conditions: $15 \mathrm{~min}$ at $37^{\circ} \mathrm{C}, 5 \mathrm{~s}$ at $85^{\circ} \mathrm{C}$, and $10 \mathrm{~min}$ at $4{ }^{\circ} \mathrm{C}$. All cDNA was stored at $-20^{\circ} \mathrm{C}$.

\subsection{Double-Stranded RNA (dsRNA) Synthesis}

The interference primers were subjected to biosynthesis according to the T7 RNA polymerase promoter (Table S1). The DNA templates for dsRNA synthesis were PCR-amplified using primer sets containing the T7 promoter sequence from previously mentioned cDNA. The interference primers were synthesized according to the T7 RNA polymerase promoter. Sequences of dsRNA were compared with sequences of the ecdysone signaling pathway genes in the transcriptome data. A $565 \mathrm{bp}$ fragment of the target gene (EcRA, ADK66917.1) and a $467 \mathrm{bp}$ fragment of the green fluorescent protein gene (GFP, ACY56286.1) were amplified using specific primers [42]. PCR products were analyzed on 1\% agarose gel, after which the PCR products were cloned and sequenced to confirm their identities. The resulting PCR products were then purified with a DNA cleanup kit and used as templates for in vitro transcription using the Megascript $\mathrm{T} 7$ transcription kit (Invitrogen, Carlsbad, CA, USA) according to the manufacturer's instructions. dsRNA was amplified using primers with the T7 RiboMAX ${ }^{\mathrm{TM}}$ Express RNAi system. The resulting dsRNA was dissolved in DEPC water and quantified at $260 \mathrm{~nm}$ using a spectrophotometer Nanodrop2000C (Thermo Fisher Scientific, Carlsbad, CA, USA). The diluted dsRNA was used immediately or stored in $10 \mu \mathrm{L}$ aliquot at $-80^{\circ} \mathrm{C}$.

\subsection{RNA Interference (RNAi) and Bioassays}

The one $\mu \mathrm{g}$ dsRNA was injected into L3 larva (molt $<3 \mathrm{~h}$ ) using a microsyringe (Hamilton, Reno, NV, USA). After the injection, the L3 larvae were placed into 24 orifice tissue culture plates containing the artificial diet via the drug-membrane method; $100 \mu \mathrm{L}$ of $\operatorname{MET}\left(\mathrm{LC}_{30}=21.00 \mathrm{ng} / \mathrm{cm}^{2}\right)$ was added to each orifice. The control was injected with dsGFP. All treatments contained MET-dsGFP and MET-dsEcRA. Every treatment was replicated five times with 120 larvae, for a total of 240 treated larvae. After the successful knockdown of EcRA, the mortality of S. exigua larvae to MET was calculated after $72 \mathrm{~h}$. 
We collected the surviving L3 larvae following treatment at $12 \mathrm{~h}, 24 \mathrm{~h}$, and $48 \mathrm{~h}$, and the L4 larvae following treatment at $72 \mathrm{~h}$. The larval cuticles were dissected, and total RNA extracted. The cDNA was synthesized from total RNA and stored at $-20{ }^{\circ} \mathrm{C}$.

\subsection{Expression of Ecdysone Signaling Pathway Genes Regulated by MET-dsEcRA}

In order to analyze the expression of ecdysone signaling pathway genes regulated by MET-dsEcRA, survival L4 larvae treated with MET-dsGFP or MET-dsEcRA, were collected in Section 4.7. Total RNA was extracted, cDNA was synthesized and stored at $-20^{\circ} \mathrm{C}$.

\section{9. qPCR Assays}

The expression of EcRA, USP, E74, HR3, HR4, FTZ-F1, HR38, TPS, TREA, HK, G6PI, GFAT, PGM, UAP, and CHSA were analyzed after the larvae were treated with sublethal doses of MET and MET-dsRNA via qPCR. The interference efficiency of dsEcRA was performed by qPCR from $12 \mathrm{~h}$ to $72 \mathrm{~h}$. The qPCR program was set to the following cycling conditions: $30 \mathrm{~s}$ at $95{ }^{\circ} \mathrm{C}, 40$ cycles of $5 \mathrm{~s}$ at $95{ }^{\circ} \mathrm{C}, 10 \mathrm{~s}$ at $60{ }^{\circ} \mathrm{C}$, and $25 \mathrm{~s}$ at $72{ }^{\circ} \mathrm{C}$. The reference genes were the elongation factor ( $\mathrm{SeEF}$ ) and Glyceraldehyde-3-phosphate dehydrogenase $(S e G A P D H)$ [43]. All samples were performed in triplicate. Target genes were calculated using the relative quantitative method $\left(2^{-\Delta \Delta C T}\right)$.

\subsection{Statistical Data Analysis}

All the results in the experiment were presented as the mean \pm standard error (SE). Significant differences were determined by Student's $t$-test, or one-way ANOVA, followed by a least significant difference test for mean comparison. The bioassay data were analyzed using IBM SPSS Statistics 20 (SPSS, Chicago, IL, USA). The primers were designed in Beacon Designer 7.0 software (Premier Biosoft, Palo Alto, CA, USA) and the pictures were composite using GraphPad Prism 7.0 (GraphPad Software Inc., San Diego, CA, USA).

Supplementary Materials: The following are available online at https:/ / www.mdpi.com/article/10 $.3390 /$ ijms22169080/s1.

Author Contributions: Conceptualization, Z.Z. and Y.M. (Yajie Ma); Methodology, X.M. and H.H.; Validation, all the authors; Formal Analysis, D.W. and X.S.; Investigation, all the authors; Resources, all the authors; Data Curation, all the authors; Writing-Original Draft Preparation, Z.Z., Y.M. (Yajie Ma), D.W. and X.S.; Writing-Review and Editing, all the authors; Visualization, all the authors; Supervision, X.R. and Y.M. (Yan Ma); and Project Administration, Y.M. (Yan Ma) All authors have read and agreed to the published version of the manuscript.

Funding: This research was founded by the Agricultural Science and Technology Innovation Program of Chinese Academy of Agricultural Sciences and Central Public-interest Scientific Institution Basal Research Fund (No.1610162021029).

Institutional Review Board Statement: Not applicable.

Informed Consent Statement: Informed consent was obtained from all subjects involved in the study.

Data Availability Statement: The raw RNA-seq data are available in the National Center for Biotechnology Information (NCBI) under SRA accession number: PRJNA705838.

Conflicts of Interest: The authors declare no conflict of interest.

\section{References}

1. Lai, T.; Li, J.; Su, J. Monitoring of beet armyworm Spodoptera exigua (Lepidoptera: Noctuidae) resistance to chlorantraniliprole in China. Pestic. Biochem. Physiol. 2011, 101, 198-205. [CrossRef]

2. Ishtiaq, M.; Saleem, M.A.; Razaq, M. Monitoring of resistance in Spodoptera exigua (Lepidoptera: Noctuidae) from four districts of the Southern Punjab, Pakistan to four conventional and six new chemistry insecticides. Crop. Prot. 2012, 33, 13-20. [CrossRef]

3. Su, J.; Sun, X.-X. High level of metaflumizone resistance and multiple insecticide resistance in field populations of Spodoptera exigua (Lepidoptera: Noctuidae) in Guangdong Province, China. Crop. Prot. 2014, 61, 58-63. [CrossRef]

4. Ahmad, M.; Arif, M.I. Resistance of beet armyworm Spodoptera exigua (Lepidoptera: Noctuidae) to endosulfan, organophosphorus and pyrethroid insecticides in Pakistan. Crop. Prot. 2010, 29, 1428-1433. [CrossRef] 
5. Kumar, M.S.; Kabra, A.N.; Min, B.; El-Dalatony, M.M.; Xiong, J.; Thajuddin, N.; Lee, D.S.; Jeon, B.H. Insecticides induced biochemical changes in freshwater microalga Chlamydomonas mexicana. Environ. Sci. Pollut. Res. Int. 2016, 23, 1091-1099. [CrossRef] [PubMed]

6. Osorio, A.; Martinez, A.M.; Schneider, M.I.; Diaz, O.; Corrales, J.L.; Aviles, M.C.; Smagghe, G.; Pineda, S. Monitoring of beet armyworm resistance to spinosad and methoxyfenozide in Mexico. Pest. Manag. Sci. 2008, 64, 1001-1007. [CrossRef] [PubMed]

7. Jiang, B.; Guo, B.; Cui, J.; Dong, Y.; Cui, L.; Zhang, L.; Yang, Q.; Yang, X. New lead discovery of insect growth regulators based on the scaffold hopping strategy. Bioorg. Med. Chem. Lett. 2020, 30, 127500. [CrossRef]

8. Petryk, A.; Warren, J.T.; Marqués, G.; Jarcho, M.P.; Gilbert, L.I.; Kahler, J.; Parvy, J.P.; Li, Y.; Dauphin-Villemant, C.; O'Connor, M.B. Shade is the Drosophila P450 enzyme that mediates the hydroxylation of ecdysone to the steroid insect molting hormone 20-hydroxyecdysone. Proc. Natl. Acad. Sci. USA 2003, 100, 13773-13778. [CrossRef] [PubMed]

9. Guo, W.-C.; Liu, X.-P.; Fu, K.-Y.; Shi, J.-F.; Lü, F.-G.; Li, G.Q. Functions of nuclear receptor HR3 during larval-pupal molting in Leptinotarsa decemlineata (Say) revealed by in vivo RNA interference. Insect Biochem. Mol. Biol. 2015, 63, 22-33. [CrossRef]

10. Xu, Q.-Y.; Meng, Q.; Deng, P.; Guo, W.-C.; Li, G.Q. Leptinotarsa hormone receptor 4 (HR4) tunes ecdysteroidogenesis and mediates 20-hydroxyecdysone signaling during larval-pupal metamorphosis. Insect Biochem. Mol. Biol. 2017, 94, 50-60. [CrossRef]

11. Liu, X.-P.; Fu, K.-Y.; Lü, F.-G.; Meng, Q.; Guo, W.-C.; Li, G.Q. Involvement of FTZ-F1 in the regulation of pupation in Leptinotarsa decemlineata (Say). Insect Biochem. Mol. Biol. 2014, 55, 51-60. [CrossRef]

12. Yogindran, S.; Rajam, M.V. Artificial miRNA-mediated silencing of ecdysone receptor (EcR) affects larval development and oogenesis in Helicoverpa armigera. Insect Biochem. Mol. Biol. 2016, 77, 21-30. [CrossRef]

13. Chen, J.; Tang, B.; Chen, H.; Yao, Q.; Huang, X.; Chen, J.; Zhang, D.; Zhang, W. Different functions of the insect soluble and membrane-bound trehalase genes in chitin biosynthesis revealed by RNA interference. PLoS ONE 2010, 5, e10133. [CrossRef]

14. Yao, Q.; Zhang, D.; Tang, B.; Chen, J.; Chen, J.; Lu, L.; Zhang, W. Identification of 20-hydroxyecdysone late-response genes in the chitin biosynthesis pathway. PLoS ONE 2010, 5, e14058. [CrossRef]

15. Chen, J.; Li, T.; Pang, R. miR-2703 regulates the chitin biosynthesis pathway by targeting chitin synthase 1a in Nilaparvata lugens. Insect Mol. Biol. 2020, 29, 38-47. [CrossRef] [PubMed]

16. Yang, M.; Zhao, L.; Shen, Q.; Xie, G.; Wang, S.; Tang, B. Knockdown of two trehalose-6-phosphate synthases severely affects chitin metabolism gene expression in the brown planthopper Nilaparvata lugens. Pest. Manag. Sci. 2017, 73, 206-216. [CrossRef]

17. Gu, S.H.; Lin, J.L.; Lin, P.L. PTTH-stimulated ERK phosphorylation in prothoracic glands of the silkworm, Bombyx mori: Role of $\mathrm{Ca}(2+) /$ calmodulin and receptor tyrosine kinase. J. Insect Physiol. 2010, 56, 93-101. [CrossRef]

18. Scieuzo, C.; Nardiello, M.; Salvia, R.; Pezzi, M.; Chicca, M.; Leis, M.; Bufo, S.A.; Vinson, S.B.; Rao, A.; Vogel, H.; et al. Ecdysteroidogenesis and development in Heliothis virescens (Lepidoptera: Noctuidae): Focus on PTTH-stimulated pathways. J. Insect Physiol. 2018, 107, 57-67. [CrossRef] [PubMed]

19. Rybczynski, R.; Bell Sc Fau-Gilbert, L.I.; Gilbert, L.I. Activation of an extracellular signal-regulated kinase (ERK) by the insect prothoracicotropic hormone. Mol. Cell. Endocrinol. 2001, 184, 1-11. [CrossRef]

20. Yao, Q.; Xu, S.; Dong, Y.; Quan, L.; Chen, B. Ecdysone Receptor (EcR) and Ultraspiracle Protein (USP) Genes From Conopomorpha sinensis Bradley Eggs: Identification and Expression in Response to Insecticides. Front. Physiol. 2020, 11, 851. [CrossRef]

21. Smagghe, G.; Böhm, G.-A.; Richter, K.; Degheele, D. Effect of Nonsteroidal Ecdysteroid Agonists on Ecdysteroid Titer in Spodoptera exigua and Leptinotarsa decemlineata. J. Insect Physiol. 1995, 41, 971-974. [CrossRef]

22. Sun, X.; Song, Q.; Barrett, B. Effects of ecdysone agonists on the expression of EcR, USP and other specific proteins in the ovaries of the codling moth (Cydia pomonella L.). Insect Biochem. Mol. Biol. 2003, 33, 829-840. [CrossRef]

23. Chen, J.; Jiang, W.; Hu, H.; Ma, X.; Li, Q.; Song, X.; Ren, X.; Ma, Y. Joint toxicity of methoxyfenozide and lufenuron on larvae of Spodoptera exigua Hübner (Lepidoptera: Noctuidae). J. Asia-Pac. Entomol. 2019, 22, 795-801. [CrossRef]

24. Aguirre, O.U.; Martinez, A.M.; Campos-Garcia, J.; Hernandez, L.A.; Figueroa, J.I.; Lobit, P.; Vinuela, E.; Chavarrieta, J.M.; Smagghe, G.; Pineda, S. Foliar persistence and residual activity of methoxyfenozide against beet armyworm (Lepidoptera: Noctuidae). Insect Sci. 2013, 20, 734-742. [CrossRef] [PubMed]

25. Mosallanejad, H.; Soin, T.; Smagghe, G. Selection for resistance to methoxyfenozide and 20-hydroxyecdysone in cells of the beet armyworm, Spodoptera exigua. Arch. Insect Biochem. Physiol. 2008, 67, 36-49. [CrossRef] [PubMed]

26. Do Nascimento, A.R.; Fresia, P.; Consoli, F.L.; Omoto, C. Comparative transcriptome analysis of lufenuron-resistant and susceptible strains of Spodoptera frugiperda (Lepidoptera: Noctuidae). BMC Genom. 2015, 16, 985. [CrossRef] [PubMed]

27. Giraudo, M.; Audant, P.; Feyereisen, R.; Le Goff, G. Nuclear receptors HR96 and ultraspiracle from the fall armyworm (Spodoptera frugiperda), developmental expression and induction by xenobiotics. J. Insect Physiol. 2013, 59, 560-568. [CrossRef] [PubMed]

28. Sun, S.Q.; Wang, N.M.; Li, J.J.; Jin, M.H.; Xue, C.B. Reduced fecundity and regulation of reproductive factors in flubendiamideresistant strains of Plutella xylostella. Pestic. Biochem. Physiol. 2020, 169, 104668. [CrossRef]

29. Tang, B.; Yang, M.; Shen, Q.; Xu, Y.; Wang, H.; Wang, S. Suppressing the activity of trehalase with validamycin disrupts the trehalose and chitin biosynthesis pathways in the rice brown planthopper, Nilaparvata lugens. Pestic. Biochem. Physiol. 2017, 137, 81-90. [CrossRef]

30. Chen, J.; Lu, Z.; Li, M.; Mao, T.; Wang, H.; Li, F.; Sun, H.; Dai, M.; Ye, W.; Li, B. The mechanism of sublethal chlorantraniliprole exposure causing silkworm pupation metamorphosis defects. Pest. Manag. Sci. 2020, 76, 2838-2845. [CrossRef]

31. Zhang, W.; Ma, L.; Liu, X.; Peng, Y.; Liang, G.; Xiao, H. Dissecting the roles of FTZ-F1 in larval molting and pupation, and the sublethal effects of methoxyfenozide on Helicoverpa armigera. Pest. Manag. Sci. 2021, 77, 1328-1338. [CrossRef] [PubMed] 
32. Shi, J.F.; Fu, J.; Mu, L.L.; Guo, W.C.; Li, G.Q. Two Leptinotarsa uridine diphosphate N-acetylglucosamine pyrophosphorylases are specialized for chitin synthesis in larval epidermal cuticle and midgut peritrophic matrix. Insect Biochem. Mol. Biol. 2016, 68, 1-12. [CrossRef] [PubMed]

33. Li, X.; Ren, X.; Liu, Y.; Smagghe, G.; Liang, P.; Gao, X. MiR-189942 regulates fufenozide susceptibility by modulating ecdysone receptor isoform B in Plutella xylostella (L.). Pestic. Biochem. Physiol. 2020, 163, 235-240. [CrossRef]

34. Xu, Q.-Y.; Deng, P.; Zhang, Q.; Li, A.; Fu, K.-Y.; Guo, W.-C.; Li, G.Q. Ecdysone receptor isoforms play distinct roles in larval-pupaladult transition in Leptinotarsa decemlineata. Insect Sci. 2019, 27, 487-499. [CrossRef]

35. Wu, J.J.; Mu, L.L.; Kang, W.N.; Ze, L.J.; Shen, C.H.; Jin, L.; Anjum, A.A.; Li, G.Q. RNA interference targeting ecdysone receptor blocks the larval-pupal transition in Henosepilachna vigintioctopunctata. Insect Sci. 2021, 28, 419-429. [CrossRef]

36. Lenaerts, C.; Van Wielendaele, P.; Peeters, P.; Vanden Broeck, J.; Marchal, E. Ecdysteroid signalling components in metamorphosis and development of the desert locust, Schistocerca gregaria. Insect Biochem. Mol. Biol. 2016, 75, 10-23. [CrossRef]

37. Shen, C.; Xu, Q.-Y.; Mu, L.-L.; Fu, K.-Y.; Guo, W.-C.; Li, G.Q. Involvement of Leptinotarsa hormone receptor 38 in the larval-pupal transition. Gene 2020, 751, 144779. [CrossRef] [PubMed]

38. Xu, Q.-Y.; Deng, P.; Li, A.; Zhang, Q.; Mu, L.-L.; Fu, K.-Y.; Guo, W.-C.; Li, G.Q. Functional characterization of ultraspiracle in Leptinotarsa decemlineata using RNA interference assay. Insect Mol. Biol. 2019, 28, 676-688. [CrossRef]

39. Ren, X.-L.; Chen, R.-R.; Zhang, Y.; Ma, Y.; Cui, J.-J.; Han, Z.-J.; Mu, L.-L.; Li, G.Q. A Spodoptera exigua Cadherin Serves as a Putative Receptor for Bacillus thuringiensis Cry1Ca Toxin and Shows Differential Enhancement of Cry1Ca and Cry1Ac Toxicity. Appl. Environ. Microbiol. 2013, 79, 5576-5583. [CrossRef]

40. Luan, J.B.; Ghanim, M.; Liu, S.S.; Czosnek, H. Silencing the ecdysone synthesis and signaling pathway genes disrupts nymphal development in the whitefly. Insect Biochem. Mol. Biol. 2013, 43, 740-746. [CrossRef] [PubMed]

41. Swevers, L.; Soin, T.; Mosallanejad, H.; Iatrou, K.; Smagghe, G. Ecdysteroid signaling in ecdysteroid-resistant cell lines from the polyphagous noctuid pest Spodoptera exigua. Insect Biochem. Mol. Biol. 2008, 38, 825-833. [CrossRef] [PubMed]

42. Wang, L.X.; Niu, C.D.; Zhang, Y.; Jia, Y.L.; Zhang, Y.J.; Zhang, Y.; Zhang, Y.Q.; Gao, C.F.; Wu, S.F. The NompC channel regulates Nilaparvata lugens proprioception and gentle-touch response. Insect Biochem. Mol. Biol. 2019, 106, 55-63. [CrossRef] [PubMed]

43. Zhu, X.; Yuan, M.; Shakeel, M.; Zhang, Y.-J.; Wang, S.; Wang, X.; Zhan, S.; Kang, T.; Li, J. Selection and Evaluation of Reference Genes for Expression Analysis Using qRT-PCR in the Beet Armyworm Spodoptera exigua (Hubner) (Lepidoptera: Noctuidae). PLoS ONE 2014, 9, e84730. [CrossRef] [PubMed] 